\title{
Research on the Operation Practice of Deliberate Democracy From the Perspective of National Governance
}

\author{
Yanyan $\mathrm{Zhu}^{1^{*}}$, Weifeng $\mathrm{Li}^{2}$, Dong Liang ${ }^{3}$ \\ ${ }^{1,2,3}$ School of Political Scinence and Law, University of Jinan, Jinan, Shandong 250022, China \\ ${ }^{*}$ Corresponding author. Email:1782231380@qq.com
}

\begin{abstract}
In recent years, China's deliberate democracy has made continuous development. It has unique advantages in promoting the integration of social relations, strengthening democratic supervision and improving decision-making efficiency. At the same time, it is of great significance to promote social development and harmony.Taking the practice of deliberatedemocracy in Jinan City as an example, this paper analyzes the advantages and disadvantages of deliberate democracy in the actual operation process in order to find a suitable way to promote the orderly implementation of deliberate democracy in local governments.
\end{abstract}

Keywords: deliberate democracy, national governance, modernization $\square$

\section{INTRODUCTION}

In recent years, with the great attention of the Party Central Committee and the joint efforts of the people of all ethnic groups in China, China's deliberate democracy has achieved great results. As an important part of the political system with Chinese characteristics, deliberate democracy has a high degree of correspondence with the modernization of national governance in the aspects of value concept, system, essential attribute and realism. And, the modernization of national governance in the new era also puts forward new requirements for deliberate democracy, which requires continuous optimization of the system structure, so as to promote the extensive and multi-level institutionalization of deliberate democracy. This paper takes the practice of deliberate democracy in Jinan City, Shandong Province as an example to explore the effectiveness and shortcomings of the local government in promoting the deliberate democracy system, in order to find a suitable path to promote the deliberate democracy government.

\section{THE PRACTICE OF DELIBERATE DEMOCRACY}

\subsection{Consultation platform}

From the perspective of modernization of national governance, deliberate democracy has an extensive and multi-level platform for practice. The important form of the people's political deliberate conference and practice platform, based on the extension of the deliberate democracy channels and platforms, including party, government, Chinese people's political deliberate conference (CPPCC), the National People's Congress and people's organizations, grass-roots consultations, formed a multi-level, diversified forms of negotiation, to expand coverage of the deliberate democracy to all levels of society, to safeguard the people's right to know, supervise, and participation.

\subsection{Deliberate subject}

The subject of deliberate democracy mainly refers to all citizens participating in the consultation process, namely. Diverse subjects of deliberate democracy actively contribute their opinions and suggestions on deliberate issues in the process of consultation, which has become an important form of promoting socialist democracy and created a new situation in which various groups have been widely involved in political life.

\subsection{Deliberate issues}

The subject of consultation, namely the object of consultation, refers to the object discussed by the subject of deliberation in the process of deliberate democracy which involves a series of major social issues related to social development. In the continuous exploration and improvement of deliberate democracy, local governments at all levels pay close attention to the scientific nature and representativeness of deliberate topics, and determine topics that are ultimately crucial to economic and social development through research and other links. 


\subsection{Deliberate process}

In the application of deliberate democracy, local governments are in accordance with scientific rules to meet the requirements of democratic centralism and collect issues concerning the development of society within the scope of the whole society through the investigation and research, and other forms, such as clear deliberate issues and content, and organize activities of consultation. Deliberate including a fruitful implementation and feedback mechanism is determined at the same time, which can promote the transformation of the deliberate results.

\subsection{Deliberate results}

The relevant proposals, important proposals and inspection reports formed during the consultation shall be supervised and implemented by the relevant bodies of the PARTY Committee and government of the CPPCC, and the consultation results shall be reported to the office of the CPPCC in writing. The CPPCC adopts a series of methods, such as holding symposiums, organizing investigations, inspections and visits, to follow up the adoption and implementation of the consultation results and ensure the implementation of the consultation results.

\section{CASE ANALYSIS}

Jinan of shandong province people's political deliberate conference in 2017, after the transition to further implement the 19 big spirits and xi general secretary "have something good to discuss" the important thoughts, give full play to its more important channel in deliberate democracy and the role of the specialized agencies, to build "Chinese people's political deliberate conference for the people, pragmatic Chinese people's political deliberate conference (CPPCC), open people's political deliberate conference" platform, expand orderly social from all walks of life to participate in democratic consultation, safeguard people's immediate interests, positive response "to discuss something good, Chinese people's political deliberate conference (CPPCC) plays" slogan, set up the new platform quancheng "consult" deliberate democracy. So far, the "consultation" in Jinan has launched more than 20 special topics, striving to seek the greatest common denominator.

\subsection{The advantages and characteristics of the "consultation" platform}

\subsubsection{Build a consultation platform with the help of party organization networks at all levels}

The deliberate democracy platform selects issues closely related to the interests of the masses and social development, organizes CPPCC members, experts, people's representatives and relevant government departments to discuss, carry out adequate communication and exchange activities, reach consensus on relevant consultation topics, and then release them to the society through media platforms.

\subsubsection{Demonstration about the people-oriented development thought in the new era}

As an important platform for deliberate democracy, the CPPCC of Jinan actively innovates in the development of deliberate democracy platform and implements the guidelines and policies of the Municipal Party Committee and the municipal government. In addition to scholars and experts, party and government officials and grassroots representatives, more than 40 CPPCC members participated in each consultation topic to conduct field research and listen to the opinions of the masses.

\subsubsection{Highlights about the advantageous role of the CPPCC in deliberate democracy}

The successful practice of Quancheng "consultation" platform fully reflects the political consultation, democratic supervision and participation in the deliberation and administration of state affairs of the CPPCC, and provides experience for local deliberate democracy practice. On the one hand, party and government departments, CPPCC, experts and scholars, and grassroots representatives can conduct in-depth exchanges and consultations on an equal footing. On the other hand, the government interacts with the public online and offline through the Internet, and extensively collects opinions and suggestions, striving to respect the wishes of the majority while accommodating the reasonable interests of the minority.

\subsubsection{Work standards for deliberate democracy should be formulated to standardize the pattern of deliberate democracy}

In order to give full play to the great advantages of deliberate democracy, "consultation" in Jinan has achieved full coverage and expanded to districts and counties. Based on this, each district and county in Jinan has formed a consultation platform of "consultation in districts and counties", and some towns and townships have also launched this consultation work, effectively promoting the construction of grassroots deliberate democracy. The Huaiyin District of Jinan issued documents and established a "consultation" platform covering areas, streets and communities. In order to promote the innovation, upgrading and flexibility of the "consultation" activities, the CPPCC has adopted various forms such as netizen 
interaction, media live broadcast and remote consultation, which have expanded its publicity and enhanced its influence.

\subsubsection{Focus on procedural construction}

The Municipal Party Committee of Jinan attaches great importance to the construction and development of the "consultation" platform in Quancheng. The results of the consultation will be sent to the Municipal Party Committee of Jinan, the municipal government and relevant municipal departments in a timely manner. The CPPCC of Jinan will organize follow-up visits, actively carry out democratic supervision and promote the implementation of the "consultation" results. Jinan Municipal Party Committee and jinan Municipal government attach great importance to the handling of the "consultation" results, and promote the quality and effectiveness of the consultation results. At the same time, the handling and implementation of the "consultation" results through a certain form of publicity.

\subsection{Some discussions about the problems of the platform}

\subsubsection{Limitations exist in the sources of negotiation topics}

The discussion topics of Jinan "consultation" platform are selected through four channels. The first one is to select the major livelihood issues from the annual government work report of China. The second kind comes from the important issues sorted out by sub-district offices and residential (village) committees. The third kind comes from the "12345" citizen service hotline, which is generally concerned and reflected by the people. The fourth is to use the Internet platform to widely solicit public opinions from the society and collect consultation topics, mainly including government web pages and WeChat public accounts, etc., so as to obtain the issues concerned by the people, especially the residents of Jinan, and involve their vital interests. Deliberate democracy itself is political participation, and its process is the process of attracting extensive participation in national governance from all sectors of society and the general public[1]. Therefore, there are still some limitations in the discussion topics obtained through the above forms, and it is still necessary to explore higher-level and broader ways to acquire topics that will boost the overall layout of social development.

\subsubsection{The specialty of deliberate subject needs to be strengthened}

In order to give full play to the important role of "consultation" platform in deliberate democracy in Jinan city, we should strengthen the professional construction of consultation subjects and absorb more professional talents. At the same time, the diversity of professional teams should be increased so that they can make suggestions on economic and social development. If we want to study deliberative democracy and thoroughly understand its growth and development process and the laws behind it, we must make a clear judgment based on China's historical filling and realistic soil[2]. In particular, they can provide professional guidance on policy and system formulation, overall urban development planning and economic development of Jinan, so as to promote the overall development within Jinan.

\subsubsection{The implementation procedures of the platform need to be improved}

Whether the opinions and suggestions formed during the consultation are adopted or not, and the actual situation of implementation after adoption, the participants in the consultation should be ensured to know. As far as the attributes of system are concerned, there are mainly two kinds: substantive system and procedural system[3].Only when the information feedback is received in a timely manner can the enthusiasm and initiative of the participants be more fully aroused. On the other hand, the outcome information of the consultation should be more transparent and open. For the groups directly related to the discussion topic and decision, the feedback information should be ensured to receive, and the opinions and suggestions of the stakeholder groups should be constantly listened to during the implementation of the outcome practice.

\subsubsection{The weak participation consciousness}

Multiple subjects capable of equal dialogue and rational consultation are the basic elements of deliberate democracy and of great significance to the modernization of national governance capacity in the transitional period[4]. In the practice of deliberate democracy, the participation consciousness of the deliberate subject is low. Consultative democracy focuses on protecting citizens' freedom of speech[5], so everyone should take part in their active role. Relative to the elite deliberate body, part of the grassroots participation main body influenced by conservative ideas, their own lack of self-consciousness and democratic consciousness in political life, can't clear its own position, can't clear interests, greatly hindered the deepened of deliberate democracy, expand citizens orderly participation in political life. In addition, the publicity of deliberate democracy in the context of national governance modernization is not high enough, and the cultivation of political participants, especially ordinary people, in terms of humanities and culture, leads to the lack of due awareness of the deliberate democracy in practice. 


\section{CONCLUSION}

Deliberate democracy from the perspective of national governance modernization emphasizes the diversity and interactivity of deliberate subjects, which requires corresponding political and cultural environment to encourage citizens to participate in political and cultural life in an active and orderly way. From the perspective of realistic political development, there are still obvious cultural features of subjects in our political life. Under the influence of traditional culture, the backward thought of official standard still restricts people's hearts, and the masses have a high dependence on government officials. Therefore, there exist many negative problems such as unclear political position, weak subjective consciousness and weak concept of rights and obligations in some groups. Under the practical platform of deliberate democracy, the masses do not have the corresponding political and cultural environment to improve their own political participation quality. The subject consciousness is also low, so it is easy to form a passive acceptance attitude in the process of participating in the deliveration. In recent years, China's socialist deliberate democracy has made continuous development. It has unique advantages in promoting the integration of social relations, strengthening democratic supervision and improving decision-making efficiency, and is of great significance to promote economic and social development and social harmony and stability. However, at present, deliberate democracy still needs to be strengthened in terms of legal authority, institutional nature and consultation subject construction, and it needs to be continuously improved to serve the modernization of national governance.

\section{ACKNOWLEDGMENT}

This work was supported by the key research project of United Front Work Department of Jinan Municipal Party Committee "Research on Deliberate Democracy from the Perspective of National Governance Modernization".

\section{REFERENCES}

[1] Bao Xinjian. Political Wisdom of Great Powers Government: New Frontier of National Governance Function through Deliberate Democracy in New Age[J]. Journal of University of Jinan(Social Science Edition)2018,28(03):24-35+157.

[2] Sun Yunjun. On Value and Rules of Socialist Consultative Democracy in the National Governance. System Journal of the Central Institute of Socialism, ,2015(04):53-57.
[3] Wang Xuejian,Yang Chang-hua. Analysis on the Values, Problems and Path of Institutionalized Consultative Democracy-From the Perspective of Modernizing National Governance[J]. Journal of Social Science of Hunan Normal University,2014,43(05):5-10.

[4] Yi Chengzhi. Deliberate Democracy, State Building and State Governance[J]. Academic Monthly, 2018,28(03):24-35+157.

[5] Ye Xiaowen,Zhang Feng. Understanding deliberative democracy from the perspective of modern state governance $[\mathrm{J}]$. Journal of the Central Institute of Socialism,2014(01):5-8. 